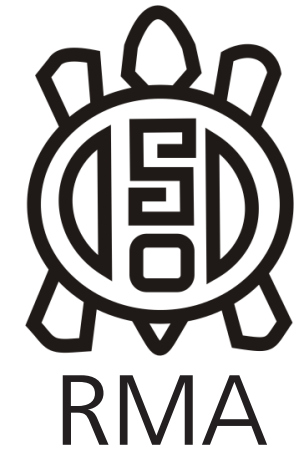

Dossier

\title{
Aportes de la tafonomía actualística al estudio arqueológico de los pseudoartefactos
}

\author{
Contributions of Actualistic Taphonomy to the Archaeological Study of \\ Pseudoartifacts
}

Karen Borrazzo*

*CONICET, Instituto Multidisciplinario de Historia y Ciencias Humanas. Facultad de Filosofía y Letras, Universidad de Buenos Aires. E-mail: kborrazzo@yahoo.com.ar

\begin{abstract}
Resumen
El creciente interés por comprender y cuantificar los efectos de los procesos posdepositacionales en la formación de los conjuntos líticos ha promovido la aplicación de abordajes tafonómicos que permitieron discutir el origen de patrones que a priori son considerados conductuales (tecnológicos, funcionales). Asimismo, esas aproximaciones tafonómicas mostraron que los diferentes contextos ambientales de una región (con su particular combinación de agentes y procesos) poseen efectos variables sobre los conjuntos líticos. Una aproximación tafonómica al entorno y su dinámica a escala local y regional permite estimar las características morfológicas y distribucionales del ruido tafonómico de fondo lítico (pseudoartefactos). En otras palabras, la incorporación sistemática de la tafonomía actualística -con sus componentes naturalista y experimental- a la investigación arqueológica contribuye a la generación de marcos de referencia que permiten refinar nuestras interpretaciones del registro lítico. Aquí se sintetizan algunos de los resultados obtenidos de la aplicación de este abordaje al estudio del sitio Casa de Piedra de Roselló (Chubut, Argentina). Se analizan y comparan los atributos morfométricos de una muestra experimental y un conjunto lítico procedente de la superficie del talud del sitio. Se concluye que el ruido tafonómico de fondo puede sobredimensionar el aporte de la materia prima local al conjunto lítico del sitio.
\end{abstract}

Palabras clave: tecnología lítica; tafonomía lítica; arqueología experimental; Casa de Piedra de Roselló, Patagonia.

\begin{abstract}
The increasing interest in understanding and quantifying the effects of postdepositional processes in the formation of lithic assemblages promoted the application of taphonomic approaches that allowed discussing the genesis of patterns considered of behavioral origin (technological, functional). Indeed, those taphonomic approaches showed that different environmental contexts within a region (with their specific combination of agent and processes) possess variable effects on lithic assemblages. Thus, a taphonomic approach to the settings of the archaeological record and to their local and regional dynamic make us aware of morphological and distributional characteristics of the taphonomic background noise (pseudoartifacts). In other words, the systematic incorporation of actualistic taphonomy-including both naturalistic and experimental components- to the archaeological research contributes to building frames of reference that allow refining our interpretations of the archaeological record. This paper presents and compares actualistic and archaeological data obtained from the application of this approach to the study of Casa de Piedra de Roselló site (Chubut, Argentina). I conclude that taphonomic background noise may inflate the representation of the local raw material in the lithic artifact assemblages recovered at the site.
\end{abstract}

Keywords: lithic technology; lithic taphonomy; experimental archaeology; Casa de Piedra de Roselló; Patagonia.

\section{Pseudoartefactos y Arqueología}

Los pseudoartefactos pueden ser definidos como litos de origen natural ${ }^{1}$ que poseen atributos de forma

1 Es importante aclarar que en el marco de la perspectiva tafonómica en que se produce este trabajo, los homininos son considerados agentes tafonómicos cuyas actividades tienen la capacidad y/o el potencial de producir pseudoartefactos (y tamaño) similares o idénticos a los exhibidos por los artefactos líticos producidos por manufactura (mediante el empleo de distintas técnicas: talla, picado,

\footnotetext{
a través de diferentes procesos (pisoteo, tránsito vehicular, arado) en el que participan distintos efectores. A los fines operativos, en este trabajo los términos "natural" y "tafonómico" son utilizados indistintamente y en oposición a "cultural" e "intencional".
} 
etc.) y/o modificados por uso humano (e.g. percutor) (Breuil y Lantier, 1951; Haynes, 1973). De acuerdo con el agente y/o proceso responsable de su producción, algunos autores han utilizado denominaciones específicas para referirse a distintos conjuntos de mímesis líticas. Así, los geofactos son aquellos pseudoartefactos originados por procesos geológicos y geomorfológicos, mientras que los zoofactos son resultado de la actividad de la fauna (e.g. Lopinot y Ray, 2007; Wisniewski et al., 2014). Por otra parte, existe una enorme variedad morfológica entre los productos generados debido a la diversidad de procesos tafonómicos capaces de fracturar y/o modelar rocas. Una aproximación tecno-morfológica (e.g. Aschero, 1975, 1983; Bordes, 1961) a la diversidad formal registrada en el universo de los pseudoartefactos nos permite detectar la existencia de un continuum que va desde "groseramente similar" hasta "idéntico" a los artefactos líticos. Las piezas más cercanas al primer extremo del continuum suelen ser fácilmente identificables puesto que su similitud con el material cultural es solo aparente y no resisten la aplicación de un protocolo para el análisis de artefactos líticos sin ser detectadas. Tales materiales se caracterizan por exhibir variantes de rasgos morfológicos no registradas en subproductos de los procesos de manufactura. Ejemplos de pseudoartefactos de esta clase son los producidos por uno o varios procesos combinados tales como haloclastismo, diaclasamiento, crioclastismo y stress térmico (Barnes, 1938; Breuil y Lantier, 1951).

Conforme nos acercamos al extremo opuesto ("zona intermedia", Carranza Elola, 2015: 7), donde los pseudoartefactos llegan a ser idénticos a algunos materiales culturales (ver debajo), las dificultades para la identificación de los pseudomorfos se incrementan al punto que un análisis tecno-morfológico, por exhaustivo que sea, resulta insuficiente para detectar su origen natural (Ellen y Muthana, 2010; van der Walt y Bradfield, 2018). Este subconjunto suele estar conformado por piezas líticas que han sido creadas o modificadas por procesos que involucran percusión y/o presión, los que pueden ser generados por agentes tafonómicos muy diversos (Barnes, 1939; van der Walt y Bradfield 2018). Obviamente, el hecho que la percusión y la presión sean los dos fenómenos más representados entre las piezas del extremo pseudoartefactual más difícil de diferenciar no es una casualidad. Por el contrario, esta convergencia morfológica solo pone de manifiesto que son estos mismos procesos los que operan en cualquier secuencia de talla y en varios usos de los instrumentos líticos (e.g. Crabtree, 1972). Desde ya, la disponibilidad de los mecanismos de percusión y presión en la naturaleza no asegura la producción de cualquier forma, secuencia técnica o cadena operativa representada en el repertorio tecnológico humano. Podemos sostener que conforme una secuencia productiva se complejiza (i.e. mayor cantidad de etapas), se estructura (i.e. ordenamiento de las etapas) y estandariza (i.e. reduce la variación en todos los aspectos), disminuyen las oportunidades para la replicación tafonómica. Así, las probabilidades de obtener un cabezal lítico tipo Cola de Pescado o Folsom, un núcleo Levallois (preferencial o recurrente) o un esferoide lítico con doble surco como efecto de uno o varios procesos tafonómicos son nulas. Sin embargo, los conjuntos artefactuales también incluyen piezas con secuencias de producción más sencillas, menos estructuradas y estandarizadas que suelen ser el componente mayoritario del registro lítico en todas las regiones del mundo. Tal es el caso de las extracciones uni, bi o multidireccionales de lascas y la formatización unifacial de filos cortos, largos o perimetrales. Por su parte, distintos estudios actualísticos abocados a la producción de pseudoartefactos han informado que piezas líticas con atributos morfológicos similares fueron obtenidas como resultado del transporte fluvial, la caída libre de rocas y el accionar de la fauna (e.g. Nash, 1993; Hosfield y Chambers, 2003; Lopinot y Ray, 2007; Carranza Elola, 2015; Fiedel, 2017).

Si bien aún no se ha generalizado en la comunidad arqueológica el reconocimiento de que los procesos tafonómicos pueden reproducir algunos de los tipos del repertorio tecnológico (e.g. Boëda et al., 2016; Fariña et al., 2014; Prentiss et al., 2016), el problema del solapamiento morfológico entre productos culturales y naturales que tomara popularidad con la discusión de los eolitos europeos ha promovido diferentes estrategias para su abordaje. Una aproximación al tema ha sido la realización de test ciegos entre especialistas en tecnología lítica para definir el origen cultural o natural de un conjunto lítico (Garvey y Mena, 2015). Subyace a esta posición el supuesto de que el conocimiento y la experticia en tecnología lítica son factores suficientes para la identificación de los pseudoartefactos. Si bien, como hemos sostenido antes en este texto, esta idea de la "suficiencia tecnológica" puede aplicar al reconocimiento de los productos de algunos procesos tafonómicos, no lo hace en otros en los que intervinieron la percusión y la presión como mecanismos de producción. Se ha sugerido que la frecuencia con que se observa la ocurrencia de estas modificaciones tafonómicas es variable y depende de factores tales como la materia prima, la energía involucrada, el ángulo de incidencia, entre otros (Barnes, 1939; Goodman, 1944). Otra de las estrategias implementadas para dirimir el origen cultural o tafonómico de piezas líticas incluyó caracterizaciones exhaustivas de los atributos 
morfológicos y métricos de los pseudoartefactos que pudieran ser empleadas de modo general para detectar su presencia en el registro lítico (e.g. Barnes, 1939, Duvall y Venner, 1979, Peacock, 1991; Guillespie et al., 2004). Sin embargo, debido a la multiplicidad de variables involucradas, estas caracterizaciones no pudieron ser generalizables a todos los contextos y requieren de calibraciones y/o evaluaciones para cada caso (e.g. Borrazzo, 2011, 2016).

En el marco de estas críticas y discusiones, el presente trabajo tiene por objeto proponer, desde una perspectiva tafonómica, modos holísticos o más integrales de evaluar el aporte del componente pseudoartefactual en los conjuntos líticos arqueológicos. Esta aproximación parte del principio de que es necesario conocer el fenómeno que se busca evaluar y para ello es indispensable emprender su estudio a fin de identificar elementos básicos tales como los procesos que los generan, los atributos morfométricos de los productos de cada proceso y las variaciones registradas en esos atributos en distintas rocas, siempre considerando el contexto ambiental específico. Para abordar el estudio de cada uno de estos ítems, una investigación actualística que incluya observaciones naturales y experimentación (tafonomía actualística ${ }^{2}$, sensu Marean, 1995) constituye la vía más adecuada (Binford, 1977, 1981; Behrensmeyer y Kidwell, 1985; GiffordGonzález, 1989; Pobiner y Braun, 2005, entre otros). Para ejemplificar, se presentan los resultados obtenidos en la primera etapa de la investigación en curso sobre la sílice microcristalina disponible en el complejo de aleros del sitio Casa de Piedra de Roselló, Aldea Beleiro, Chubut (Castro Esnal y Borrazzo, 2015; Castro Esnal et al., 2017, 2019).

\section{Materiales y métodos}

El complejo arqueológico Casa de Piedra de Roselló (CP) $\left(45.3^{\circ} \mathrm{S}, 71.2^{\circ} \mathrm{W}\right)$ está formado por tres aleros (CP1, 2 y 3) erosionados en la ignimbrita de la formación Carrenleufú (Castro Esnal et al., 2017). Las oquedades están emplazadas a unos $12 \mathrm{~m}$ sobre el nivel del valle; ambos espacios están conectados por un talud de unos $30 \mathrm{~m}$ de largo con una pendiente de 0 a $10^{\circ}$. El sustrato está compuesto por sedimentos finos con gravas principalmente derivados de la meteorización in situ de los afloramientos. La fauna autóctona y el ganado buscan frecuentemente reparo en los aleros. Las excavaciones arqueológicas en CP1 entregaron evidencias para su ocupación humana desde ca.

\footnotetext{
2 A diferencia de la tafonomía que implementa el método comparativo, en la tafonomía actualística "...the analyst actively controls the parameters of the observed process to increase understanding of the relations linking actor to trace" (Marean, 1995: 66).
}

9000 años AP y hasta tiempos históricos. Todas las capas en la estratigrafía de CP1 incluyen desechos e instrumentos en obsidiana, principalmente procedente de la fuente de Pampa del Asador, localizada a unos $270 \mathrm{~km}$ al sur del sitio (Castro Esnal et al., 2017). Sin embargo, la materia prima lítica que domina la secuencia es la sílice microcristalina (en adelante, SFC) que aparece bajo la forma de venas o nódulos en la formación Carrenleufú; porciones de buena calidad para la talla han sido observadas en bloques y muros de los aleros. En este contexto, el objetivo de los estudios de tafonomía lítica en curso es evaluar la existencia de un componente pseudoartefactual en CP y establecer su magnitud. Esta evaluación tafonómica posibilitará que las interpretaciones conductuales minimicen la interferencia del ruido tafonómico de fondo, así como contribuir al conocimiento de las condiciones bajo las cuales se formó el registro arqueológico local (Castro Esnal y Borrazzo, 2015).

La investigación sobre pseudoartefactos en Casa de Piedra de Roselló implementó los dos componentes de la tafonomía actualística. Los estudios naturalistas consistieron en observaciones de campo en contextos arqueológicos y no-arqueológicos de la localidad (Borrazzo, 2016). Los estudios experimentales exploraron dos procesos tafonómicos que las observaciones naturalistas indicaron como con mayor potencial para producir pseudoartefactos en CP: la caída de rocas y el pisoteo. En los experimentos de caída libre, tres conjuntos de clastos de SFC $(\mathrm{N}=6$, $\mathrm{N}=10, \mathrm{~N}=6$ ) fueron depositados sobre una superficie lisa (30 por $30 \mathrm{~cm}$ en cada caso) con un sustrato limoarcilloso seco; posteriormente, un nódulo de SFC (peso: $222,4 \mathrm{~g}$ ) fue dejado caer desde 1, 2, y $3 \mathrm{~m}$ de altura (Figura 1A, B y F). Los efectos de los impactos fueron registrados sobre los especímenes depositados. Por su parte, los experimentos de pisoteo fueron realizados por un individuo de $60 \mathrm{~kg}$ con calzado de suela de cuero blando. Las piezas experimentales ( $\mathrm{N}=42$ lascas) fueron manufacturadas por talla sobre nódulos de SFC. Se realizaron dos eventos de pisoteo: uno sobre sustrato blando (sedimentos limo-arcillosos en estado seco) y otro duro (empedrado); en ambos casos las pistas midieron de 1,5 por $1,5 \mathrm{~m}$. Cada experimento contó con cuatro series de pisoteo (entre 70 y 80 pasadas por cada serie de 10 minutos) (Figura 1L-P). Los especímenes experimentales de todos los sets fueron posteriormente analizados tecnomorfológicamente (Aschero, 1975, 1983) y comparados con una muestra de artefactos líticos de SFC ( $N=126)$ recolectada en la superficie del talud de CP por medio de seis transectas perpendiculares a la pendiente de 25 por $5 \mathrm{~m}$ cada una (superficie total muestreada $750 \mathrm{~m}^{2} ; \mathrm{N}=151 ; 0,2013$ artefactos $/ \mathrm{m}^{2}$ ) (Figura 1Q-Y). 

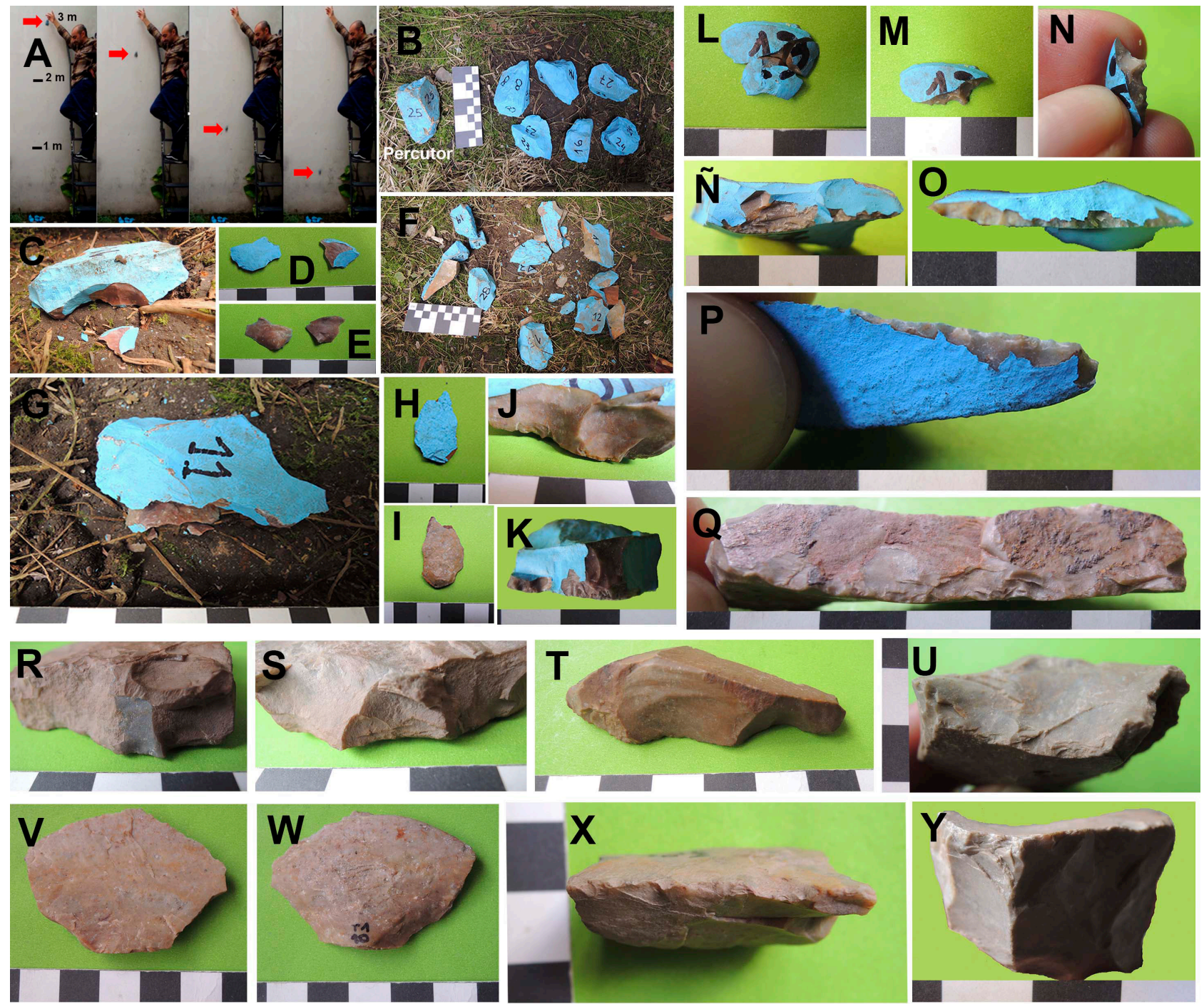

Figura 1. Especímenes líticos de SFC analizados en este estudio. A-K: Experimento 1: impacto de caída libre. A) Proceso experimental, lanzamiento desde $3 \mathrm{~m}$ de altura. B) Piezas experimentales junto al clasto de SFC utilizado para impactarlas. C y G) Lascas extraídas por un impacto desde $1 \mathrm{~m}$ de altura junto a las piezas lascadas. D y E) Cara dorsal y ventral de dos lascas extraídas por impactos desde $1 \mathrm{~m}$ de altura. F) Conjunto experimental luego de 100 impactos desde $3 \mathrm{~m}$ de altura. H e I) Cara dorsal y ventral de una lasca extraída por impacto desde $1 \mathrm{~m}$ de altura. J y K) Piezas lascadas por impactos desde $2 \mathrm{~m}$. L-P: Experimento 2 (pisoteo). Piezas experimentales luego de 40 minutos de pisoteo sobre sustrato duro. Q-Y: Piezas recolectadas en la superficie del talud de CP1.

Figure 1. Lithic SFC specimens analyzed in this study. A-K: Experiment 1 (rockfall). A) Experimental procedure, rock free falling from $3 \mathrm{~m}$ high. B) Experimental pieces and the rock used as the free falling rock in the experiments. C \& G) Flakes removed by a $1 \mathrm{~m}$ high impact lying by the flaked experimental artifacts. D \& E) Dorsal and ventral sides of two flakes removed by $1 \mathrm{~m}$ high impacts. F) Experimental assemblage after 100 impacts from $3 \mathrm{~m}$ high. $\mathrm{H} \& \mathrm{I}$ ) Dorsal and ventral side of a flake removed by a $1 \mathrm{~m}$ high impact. J \& K) Pieces flaked by $2 \mathrm{~m}$ high impacts. L-P: Experiment 2 (trampling). Experimental pieces after 40 minute trampling on hard substrate. Q-Y: Lithic specimens collected from CP1 talus surface.

\section{Resultados experimentales}

Los impactos desde las tres alturas produjeron melladuras, (pseudo) retoques (i.e. extracción de pequeñas lascas) sobre los nódulos de SFC depositados y también destacaron lascas $>2 \mathrm{~cm}(\mathrm{~N}=8, \mathrm{~N}=8$ y $\mathrm{N}=9$ extracciones a 1, 2 y $3 \mathrm{~m}$, respectivamente). Luego de 483 impactos (283³ lanzamientos desde $1 \mathrm{~m}, 100$

3 Los lanzamientos desde $1 \mathrm{~m}$ de altura se realizaron lanzamientos desde $2 \mathrm{~m}$ y 100 lanzamientos desde

sobre piezas dispuestas individualmente y el número de lanzamientos estuvo condicionado por la ocurrencia de la fractura del espécimen impactado. La fractura ocurrió en el $5^{\circ}, 12^{\circ}, 13^{\circ}, 29^{\circ}, 38^{\circ}$ y $174^{\circ}$ lanzamiento desde $1 \mathrm{~m}$. En el caso de los lanzamientos desde 2 y $3 \mathrm{~m}$ de altura, dada la imposibilidad de replicar el método por la dificultad de lograr que la roca arrojada desde esas alturas impactara la pieza depositada sobre el sustrato, se procedió a disponer grupos de piezas y realizar tantos lanzamientos como fuera necesario para alcanzar $\mathrm{N}=100$ impactos efectivos, siempre utilizando el 
$3 \mathrm{~m}$ ), la muestra original (22 nódulos) se transformó en 98 especímenes (15 corresponden a nódulos y/o fragmentos sin modificar). Los pseudomorfos ( $\mathrm{N}=83$ ) obtenidos en los experimentos de caída de rocas incluyeron lascas $(\mathrm{N}=57>5 \mathrm{~cm})$ y

desechos indiferenciados $(\mathrm{N}=62)$, artefactos formatizados $(\mathrm{N}=10)$ y núcleos $(\mathrm{N}=6)$ (Figura 1C-K).

El pisoteo de las lascas produjo mímicas de artefactos formatizados ( $\mathrm{N}=10$ ) (Figura 1L-P). Su frecuencia fue elevada solo en el sustrato duro $(45,45 \%)$. Los filos $(\mathrm{N}=12)$ obtenidos por pisoteo sobre sustrato duro fueron: muescas $(\mathrm{N}=4)$, filos largos retocados (cuchillos y raederas, $\mathrm{N}=3$ ) y filos restringidos (RBO y cortante, $\mathrm{N}=5$ ). Las lascas producidas por pisoteo fueron en todos los casos pequeñas $(\leq 5 \mathrm{~mm})$. La ocurrencia de fracturas fue alta en ambas pistas ( $55 \%$ en sustrato blando y $63,64 \%$ en sustrato duro).

\section{Comparación con la muestra del talud de $\mathrm{CP}$}

Las lascas del experimento de caída de rocas $(\mathrm{N}=57)$ exhiben un número menor de negativos de lascados en su cara dorsal con respecto a la muestra del talud $(\mathrm{N}=72)$. Solo los especímenes del talud registraron cuatro a seis negativos de lascados (Tabla 1). Las lascas experimentales enteras $(\mathrm{N}=34)$ presentan valores menores para el largo (media: 14,56 vs $36,18 \mathrm{~mm}$; mediana: 12,15 vs $34,75 \mathrm{~mm}$ ) y ancho (media: 16,35 vs $31,61 \mathrm{~mm}$; mediana: 13,75 vs 30 $\mathrm{mm}$ ) con respeto a las lascas enteras recolectadas en la superficie del talud de $\mathrm{CP}(\mathrm{N}=66)$. Además, todo el rango de valores para largo y ancho registrados entre los especímenes experimentales está incluido en los valores exhibidos por la muestra del talud. No se registraron diferencias estadísticamente significativas entre las medianas de la razón longitud:ancho de las lascas experimentales y del talud $(U=2048, p=0,8511)$. Las lascas experimentales presentan terminaciones agudas $(63,16 \%$, $\mathrm{N}=36)$, sobrepasadas $(10,53 \%, \mathrm{~N}=$
Tabla 1. Composición tecno-tipológica del conjunto lítico recolectado en el talud de CP y los pseudomorfos experimentales producidos por caída libre (desechos, artefactos formatizados y núcleos) y pisoteo (artefactos formatizados).

*Corresponden a piezas que también registran (pseudo) artefactos formatizados.

Table 1. Techno-typological composition of the lithic assemblage collected from CP talus and experimental pseudomorphs obtained by rock falling (debitage, tools and cores) and trampling (only tools).

*These specimens also exhibit pseudo-tools.

\begin{tabular}{|c|c|c|c|}
\hline & Talud & $\begin{array}{c}\text { Exp. } \\
\text { Caída libre }\end{array}$ & $\begin{array}{l}\text { Exp. } \\
\text { Pisoteo }\end{array}$ \\
\hline Desechos & 72 & 62 & - \\
\hline -Desechos indet & 6 & 5 & - \\
\hline -Lascas & 66 & 57 & - \\
\hline NEGATIVOS DE LASCADOS & & & - \\
\hline 0 & 12 & 42 & - \\
\hline 1 & 18 & 18 & - \\
\hline 2 & 20 & 1 & - \\
\hline 3 & 8 & 1 & - \\
\hline 4 & 8 & 0 & - \\
\hline 5 & 3 & 0 & - \\
\hline 6 & 1 & 0 & - \\
\hline TALONES & & & - \\
\hline Natural & 20 & 26 & - \\
\hline Liso & 20 & 12 & - \\
\hline Diedro & 9 & 1 & - \\
\hline Estallado & 3 & 2 & - \\
\hline Filiforme & 3 & 1 & - \\
\hline TERMINACIONES & & & - \\
\hline Aguda & 34 & 36 & - \\
\hline En charnela & 6 & 4 & - \\
\hline Quebrada & 5 & 0 & - \\
\hline Sobrepasada & 3 & 6 & - \\
\hline Artefactos formatizados & 43 & 10 & 10 \\
\hline Nfilos & 58 & 13 & 12 \\
\hline Raedera & 14 & 1 & 2 \\
\hline Muesca & 13 & 2 & 4 \\
\hline Raspador & 7 & 1 & 0 \\
\hline Filo retocado indeterminado & 6 & 7 & 0 \\
\hline Cuchillo & 5 & 1 & 1 \\
\hline Cepillo & 4 & 0 & 0 \\
\hline Denticulado & 3 & 1 & 0 \\
\hline Cortante & 1 & 0 & 2 \\
\hline Punta burilante & 1 & 0 & 0 \\
\hline $\mathrm{RBO}$ & 1 & 0 & 3 \\
\hline Indet. & 3 & 0 & 0 \\
\hline $\begin{array}{l}\text { Filos naturales con rastros } \\
\text { complementarios }\end{array}$ & 3 & $2^{*}$ & 0 \\
\hline Núcleos & 8 & 6 & - \\
\hline Totales & 126 & 88 & 10 \\
\hline
\end{tabular}

mismo clasto como 'percutor'. 
6) y en charnela $(7,02 \%, N=4$; Tabla 1$)$. Por su parte, las terminaciones agudas son las más frecuentes $(69,39 \%$, $\mathrm{N}=34$ ) entre las lascas del talud, seguidas por las terminaciones en charnela $(12,24 \%, N=6)$, quebrada $(12,20 \%, N=5)$ y sobrepasada $(6,12 \%, N=3$; Tabla 1$)$. Los talones corticales y lisos son los más frecuentes en el conjunto experimental $(61,90 \%, \mathrm{~N}=26$ y $28,57 \%$; $\mathrm{N}=12)$ y del talud $(35,71 \%, \mathrm{~N}=20$ y $35,71 \%, \mathrm{~N}=20)$. Las plataformas estalladas están igualmente representadas en ambos conjuntos (ca. $5 \%, \mathrm{~N}=2$ y N=3; Tabla 1). Los talones diedros muestran diferentes frecuencias en las muestras: mientras están bien representados en el conjunto del talud $(16,07 \%, N=9)$, su presencia es escasa entre las piezas experimentales $(2,38 \%$, $\mathrm{N}=1$ ) (Tabla 1). Los talones filiformes por su parte son escasos en ambos conjuntos (5,36 y 2,38\%, N=3 y $\mathrm{N}=1$, respectivamente).

En cuanto a los artefactos formatizados (Tabla 1), se observó que la muestra experimental (en este caso compuesta por piezas producidas por impacto y pisoteo, $\mathrm{N}=10$ en cada caso) y del talud comparten los principales grupos tipológicos representados: raedera, muesca y cuchillo (Tabla 1).

\section{Discusión y Conclusiones}

Los resultados presentados muestran la existencia de equifinalidad en los productos de actividades de talla antrópica y los procesos tafonómicos de caída libre y pisoteo sobre SFC. Se observó que las lascas tafonómicas -como aquellas producidas en nuestros experimentos de caída de rocas- no exhiben atributos que permitan diferenciarlas de las lascas s.s. (Aschero 1975) y tampoco habilitan su identificación en la muestra procedente del talud de CP. Como fuera señalado por otros investigadores, el presente estudio sugiere que las diferencias entre las lascas experimentales y aquellas del talud que fueron recolectadas como muestras arqueológicas son de naturaleza más cuantitativa que cualitativa (e.g. Mason, 1965, Duvall y Venner, 1979, van der Walt y Bradsfield, 2018). Es decir que las diferencias detectadas entre ambas muestras no se deben a la presencia de rasgos o tipos distintos sino fundamentalmente a variaciones en los valores exhibidos por algunos atributos cuantitativos (longitud, ancho, cantidad de negativos de lascados) o la frecuencia relativa de tipos morfológicos en cada conjunto. Los datos experimentales sugieren que este solapamiento morfométrico ubica potencialmente a ca. $68 \%$ de las lascas de SFC del conjunto del talud de CP1 como contribución pseudoartefactual. Los nuevos estudios necesariamente deberán aumentar el tamaño de la muestra experimental utilizada y ajustar el peso y frecuencia de la caída libre de rocas que tuvo y tiene lugar en $\mathrm{CP}$, diferenciando lo que ocurre dentro del alero y sobre el talud; en este último sector, se adicionan otros movimientos luego de la caída libre (ver Dorren 2003). Cabe mencionar que durante el muestreo del talud se registró la presencia de nódulos y bloques de SFC de tamaños mayores a los utilizados en nuestros experimentos, lo que sugiere la ocurrencia en el sitio de caídas que involucraron mayor energía. Por lo tanto, su frecuencia y efectos necesitan ser evaluados.

La aplicación de un enfoque tafonómico ofrece una vía más confiable para el estudio del ruido tafonómico de fondo lítico, siendo necesaria la integración de abordajes tanto tafonómicos como tecnomorfológicos. Los resultados obtenidos sugieren que debemos aprender de los contextos no-arqueológicos y los procesos allí operando para alcanzar una mayor comprensión del registro arqueológico. El reconocimiento de la existencia de situaciones de equifinalidad como la aquí ilustrada constituye un estímulo para el desarrollo de herramientas arqueológicas que permitan caracterizar los patrones tafonómicos específicos a fin de acotar la equifinalidad artefacto-pseudoartefacto de cada caso (Binford, 2001; Lyman, 2004). Ese es el objetivo de las siguientes etapas de esta investigación tafonómica.

Boedo, $1^{\circ}$ de agosto de 2019.

\section{Agradecimientos}

A los evaluadores, por sus valiosos comentarios y sugerencias. Las investigaciones presentadas en este trabajo se desarrollan en el marco de la CIC-CONICET, los proyectos PICT2014-2061 y PICT2015-2141 del FONCyT-ANCyT y el Programa de posdoctorado en Ciencias Humanas y Sociales de la FFyL-UBA.

\section{Referencias citadas}

Aschero, C.A. (1975). Ensayo para una clasificación morfológica de artefactos líticos aplicada a estudios tipológicos comparativos. Buenos Aires: CONICET. Ms.

Aschero, C.A. (1983). Ensayo para una clasificación morfológica de artefactos líticos aplicada a estudios tipológicos comparativos. Apéndices A-C. Revisión. Buenos Aires: Cátedra de Ergología y Tecnología, FFyL-UBA. Ms.

Balirán, C. (2014). Trampling, taphonomy, and experiments with lithic artifacts in the southeastern Baguales Range (Santa Cruz, Argentina). Intersecciones en Antropología, 15(3), 85-95.

Barnes, A. (1939). The differences between natural and human flaking on prehistoric flint implements. American Anthropologist, 41(1), 99-112.

Behrensmeyer, A.K. y S.M. Kidwell (1985). Taphonomy's 
Contributions to Paleobiology. Paleobiology, 11(1), 105-119.

Binford, L.R. (1977) General Introduction. En L.R. Binford (ed.), For Theory Building in Archaeology (pp. 1-13). New York: Academic Press.

Binford, L.R. (1981). Bones: Ancient Men and Modern Myths. New York: Academic Press.

Binford, L.R. (2001). Where do research questions come from? American Antiquity, 66, 669-78.

Boëda, E., R. Rocca, A. Da Costa, M. Fontugne, C. Hatté, I. Clemente-Conte, J.C. Santos, L. Lucas, G. Felice, A. Lourdeau, X Villagran, M. Gluchy, M.P. Ramos, S. Viana, C. Lahaye, N. Guidon, C. Griggo, M. Pino, A.M. Pessis, C. Borges y B. Gato (2016). New Data on a Pleistocene Archaeological Sequence in South America: Toca do Sítio do Meio, Piauí, Brazil. PaleoAmerica, 2:4, 286-302.

Bordes, F. (1961). Typologie du Paleolithique Ancien et Moyen. Impriméries. Burdeos: Delmas.

Borrazzo, K. (2011). Tafonomía lítica y pseudoartefactos: el caso de la península El Páramo (Tierra del Fuego, Argentina). Intersecciones en Antropología, 12, 155167.

Borrazzo, K. (2016) Lithic Taphonomy in desert environments: contributions from Fuego-Patagonia (Argentina). Quaternary International, 422,18-29.

Brueil, H. y R. Lantier (1951). Les hommes de la Pierre ancienne (Paléolithique et Mésolithique). París: Payot.

Carranza Elola, J.J. (2015) Tecnología y tafonomía lítica del sitio quebrada Quereo: metodología para abordar conjuntos líticos ambiguos del Pleistoceno tardío en la costa de Los Vilos. Memoria para optar al título profesional de Arqueólogo. Santiago: Universidad de Chile.

Castro Esnal, A. y K. Borrazzo (2015). Estudios de tecnología y tafonomía lítica en el sitio Casa de Piedra (Aldea Beleiro, Sudoeste de Chubut). PICT2015-2141. Buenos Aires: ANPCyT. Ms.

Castro Esnal, A, M.L. Casanueva, K. Borrazzo, G.L. L'Heureux, L. Gutiérrez, M. Campos, F. Ronco y C.B. Pérez de Micou (2019). Sitio Casa de Piedra de Roselló (SO Chubut, Patagonia, Argentina): Avances recientes y nuevas líneas de investigación. Libro de Resúmenes del XX Congreso Nacional de Arqueología Argentina (pp. 301-305). Córdoba: Universidad Nacional de Córdoba.

Castro Esnal, A., C.B. Pérez de Micou, y M.L. Casanueva (2017). Early Holocene Occupation of the
Forest-Steppe Ecotone of Southern South America: Evidence from Casa de Piedra de Roselló Cave (Chubut, Patagonia Argentina). Paleoamerica. doi: 10.1080/20555563.2017.1330102

Crabtree, D. (1972). An Introduction to Flintworking. Occasional Papers 28. Pocatello: Idaho State University Museum.

Dorren, L.K. (2003). A review of rockfall mechanics and modeling approaches. Prog Phys Geogr, 27(1), 69-87.

Duvall, J.G. y W.T. Venner (1979). A Statistical Analysis of the Lithics from the Calico Site 5BCM 1500A), California. Journal of Field Archaeology, 9, 455-462.

Ellen, R. y A. Muthana (2010). Classifying 'Eoliths': How Cultural Cognition Featured in Arguments Surrounding Claims for the Earliest Human Artifacts as these Developed Between 1880 and 1900. Journal of Cognition and Culture, 10, 341-375.

Fariña, R.A., P.S. Tambusso, L. Varela, A. Czerwonogora, M. Di Giacomo, M. Musso, R. Bracco y A. Gascue (2014). Arroyo del Vizcaíno, Uruguay: a fossil-rich 30-ka-old megafaunal locality with cut-marked bones. Proceedings of the Royal Society B (Biological Sciences), 281, 20132211. (doi:10.1098/rspb.2013.2211).

Fiedel, S.J. (2017). Did Monkeys Make the Pre-Clovis Pebble Tools of Northeastern Brazil? PaleoAmerica, $3(1), 6-12$

Gifford-Gonzalez, D. (1989). Ethnographic analogues for interpreting modified bones: some cases from East Africa. En Bonnichsen R. y M. Sorg M (eds.) Bone modification (pp. 179-246). Orono: University of Maine - Institute for Quaternary Studies.

Gillespie, J.D., S. Tupakka y C. Cluney (2004). Distinguishing Between Naturally and Culturally Flaked Cobbles: A Test Case from Alberta, Canada. Geoarchaeology, 19(7), 615-633.

Haynes, V. (1973). The Calico Site: Artifacts or Geofacts? Science, 181(4097), 305-310.

Hosfield, R. y J. Chambers (2003). Flake modifications during fluvial transportation: three cautionary tales. Lithics, 24, 57-65.

Lopinot, N. y J. Ray (2007). Trampling Experiments in the Search for the Earliest Americans. American Antiquity, 72(4), 771-782.

Lyman, R.L. (2004). The Concept of Equifinality in Taphonomy. Journal of Taphonomy 2(1), 15-26.

Marean, C. (1995). Of Taphonomy and Zooarchaeology. 


\section{Evolutionary Anthropology, 4(2), 64-72.}

Mason, R.J. (1965) Makapansgat Limeworks Fractured Stone Objects and Natural Fracture in Africa. The South African Archaeological Bulletin, 20 (77), 3-16

Nash, D.T. (1993). Distinguishing Stone Artifacts from Naturefacts created by Rockfall Processes. En Goldberg, P., D.T. Nash y M.D. Petraglia (eds.), Formation Processes in Archaeological Context. Monographs in World Prehistory 17, (pp. 125-138), Madison: Prehistory Press.

Peacock, E. (1991). Distinguishing between Artifacts and Geofacts: A Test Case from Eastern England. Journal of Field Archaeology, 18 (3), 345-361.

Pobiner, B.L. y D.R. Brown (2005). Applying Actualism:
Considerations for Future Research. Journal of Taphonomy, 3(2), 57-65.

Prentiss, A.M., K.D. Barnett y M.J. Walsh (2016). The Coarse Volcanic Industry at the Río Ibáñez 6 West Site, Chilean Patagonia: assessing Geogenic versus Anthropogenic processes. Lithic Technology, 41(2), 130-138.

Van der Walt, J. y J. Bradfield (2018). The effects of heavy-duty machinery on the formation of pseudoknapping debitage in Stone Age cultural landscapes. Antiquity, 92 366, 1429-1444.

Wisniewski, A., J. Badura, T. Salamon y J. Lewandowski (2014). The alleged early palaeolithic artefacts are in reality geofacts: A revision of the site of Kończyce Wielkie 4 in the Moravian Gate, South Poland. Journal of Archaeological Science, 52, 189-203. 